\title{
Poliformat como herramienta de apoyo en la adquisición de la expresión oral en alemán
}

Daniela Gil-Salom a y María José Gómez Perales ${ }^{\text {a }}$

${ }^{a}$ Departamento de Lingüística Aplicada, Universitat Politècnica de València, e-mail: dagil@idm.upv.es; mjgomez@idm.upv.es

\begin{abstract}
How can an institutional platform such as PoliformaT support in the acquisition of a second foreign language? The aim of this paper is to show how we guided our students from different centers and belonging to various degrees of the Universitat Politècnica de València (UPV) on an essential aspect of language learning: the correct pronunciation and intonation when speaking Standard German.

Oral expression is one of the basic skills when learning a language, but at university we do not always have small groups to develop it properly. The classroom sometimes prevents us from individual monitoring of our students and they do not even find any support outside the classroom. Digital tools (in this case, the Tasks section of PoliformaT) can help overcome this deficiency and may even encourage thoughtful learning as a condition for an independent attitude towards study. We present the practical development of this process: students listen to oral texts as a reference in order to produce their own. Then they are recorded and listened again to compare with the models and evaluate themselves. These recordings can be individual and/or in small groups.
\end{abstract}

Keywords: high education, German as a foreign language, oral expression, active methodologies, technological tools

\section{Resumen}

¿Cómo puede apoyar una plataforma institucional como PoliformaT en la adquisición de una segunda lengua extranjera? El objetivo de este trabajo es dar a conocer cómo hemos orientado a nuestros alumnos de distintas titulaciones pertenecientes a varios centros de la Universitat Politècnica de València (UPV) en un aspecto indispensable de su aprendizaje: la 
pronunciación y entonación correctas cuando se expresen en lengua alemana estándar.

La expresión oral es una de las destrezas básicas en el aprendizaje de una lengua, pero en la universidad no siempre contamos con grupos reducidos para desarrollarla adecuadamente. El aula, en ocasiones, no nos permite un seguimiento individualizado de nuestros alumnos y ellos mismos tampoco encuentran fácilmente un apoyo en su aprendizaje fuera de ella. Las herramientas digitales (en este caso, el apartado de Tareas en PoliformaT) pueden ayudar a superar esta deficiencia e incluso pueden fomentar el aprendizaje reflexivo como condición para una actitud autónoma ante el estudio. Presentamos el desarrollo práctico de este proceso: los alumnos escuchan textos orales como referencia con el objetivo de producir los suyos propios. A continuación se graban y vuelven a escucharse, para comparar con con los modelos y autoevaluarse. Estas grabaciones pueden ser individuales $\mathrm{y} / \mathrm{o}$ en pequeños grupos.

Palabras clave: enseñanza superior, alemán como lengua extranjera, expresión oral, metodologías activas, recursos tecnológicos

\section{Introducción}

Según el Marco Europeo de Referencia para las lenguas, la competencia fonética, esto es, tanto la pronunciación como la entonación, son fundamentales para que el proceso comunicativo sea efectivo (2001). Sin embargo, esta importancia no ha tenido tradicionalmente un reflejo en los materiales ni en las metodologías relacionados con la enseñanza-aprendizaje de las segundas lenguas (Panušová 2007), que han visto relegado el aspecto fonético-fonológico, tanto en su vertiente receptiva como productiva, a un lugar mucho menos destacado que el de otros niveles o aspectos relativos a ellas, como el léxico o la gramática. En este sentido G. Mebus (1995) hace notar que a menudo no son los errores gramaticales precisamente los que dificultan o impiden que la comunicación se produzca, sino que con frecuencia esta imposibilidad se debe a una pronunciación y entonación incorrectas por parte del hablante de la segunda lengua (L2). Habiendo detectado la relevancia de esta destreza para una comunicación efectiva, son numerosos los recursos que a lo largo de los años, pero sobre todo desde la irrupción de las tecnologías digitales, se han desarrollado para implementar la práctica de la competencia fonética.

Es habitual que los diccionarios bilingües en red ofrezcan la posibilidad de escuchar la palabra buscada, así como que aporten la correspondiente transcripción fonética. A este nivel fonológico también disponemos de páginas web especializadas en las que podemos 
escuchar la realización de un fonema determinado en diferentes lenguas (Cabedo 2012). Hirschfeld y Wolff (2007) al igual que Pastor (2004), sin embargo, animan a salir del ámbito aislado de la fonética y dar el salto al trabajo con diálogos, es decir, con elementos suprasegmentales de la lengua. En su caso proponen dramatizar estos diálogos teatralizándolos para concienciar al alumno/actor de la importancia de una buena pronunciación y entonación.

Para pronunciar un sonido de forma correcta, hemos de ser capaces de reconocerlo primero. Por esta razón, practicar la escucha de textos orales en la L2 con el objetivo de aprender a discriminar e identificar elementos fonéticos, es un requisito previo para ser capaz de reproducir más tarde la concatenación de secuencias de sonido, así como para imitar la melodía y el ritmo (Mebus 1995). No obstante, la capacidad para reconocer, diferenciar e identificar sonidos, así como la destreza para articularlos o para imitar la musicalidad de una lengua extranjera, -en nuestro caso, la lengua alemana por parte de discentes españolesno son iguales en todos los alumnos, ya que en unos casos la interferencia de la lengua materna, entre otros muchos aspectos, influye en mayor medida que en otros. Por ello, estamos de acuerdo con Hirschfeld y Reinke (2007) cuando destacan la importancia de la individualización en la clase de fonética.

Como veremos a lo largo de nuestro trabajo, los recursos tecnológicos de los que hoy en día dispone cualquier alumno (móvil, tableta, portátil, etc.) y la Plataforma institucional digital de la UPV, PoliformaT, favorecen este tratamiento individualizado en la orientación y tutorización del aprendizaje de las capacidades relacionadas con la adquisición de la expresión oral en lengua alemana estándar.

\section{Objetivos}

Nuestro trabajo persigue la consecución de objetivos de distinta índole, por un lado, los relacionados con una de las competencias inherentes a la asignatura: la expresión oral en alemán estándar. Y por otro, los vinculados con la metodología y procedimientos empleados para ello: uso de la herramienta de Tareas ubicada en PoliformaT.

\subsection{Objetivos de la asignatura}

La asignatura de Alemán persigue la adquisición de las cuatro destrezas comunicativas: la expresión y comprensión escritas, así como la expresión y comprensión orales. En este trabajo, nos centramos en el aprendizaje de la competencia oral en lengua alemana. Esta competencia es una realización concreta de la competencia general y transversal de la UPV, la cual pretende conseguir que todos sus egresados sean capaces de comunicarse de forma

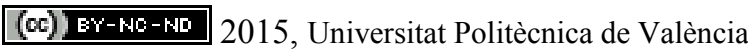


efectiva. A su vez, responde también a las competencias específicas referidas en las distintas ERT en cuanto a la capacidad de comunicación oral y escrita en lengua extranjera y la capacidad para trabajar en entornos diversos y multiculturales.

Así pues, en aras de una comunicación oral efectiva, hemos de facilitar la adquisición de la pronunciación y entonación de esta lengua extranjera, alemán stándard, como requisito previo para la consecueción de estas competencias referidas anteriormente.

\subsection{Objetivos metodológicos}

Es de todos conocido el uso cotidiano de las TIC para el aprendizaje de lenguas, tales como cursos online (Busuu, Rosetta Stone, Easy German, Deutsch Interaktiv, etc.) etc. Su popularidad y beneficios dan muestra evidente del aprovechamiento de las herramientas digitales de aprendizaje. Estos cursos, sin embargo, no se adecúan a nuestro marco académico, si bien reconocemos que pueden ser de utilidad de forma puntual y extracurricular. Nuestra función como docentes es optimizar el rendimiento de nuestros alumnos procurando detectar en todo momento las debilidades que surgen en el proceso de aprendizaje y subsanarlas.

PoliformaT representa un marco idóneo para llevar a cabo este seguimiento personalizado, por ejemplo, desde las carpetas ubicadas en el apartado de Espacio compartido para disponer de un único punto de referencia e intercambiar materiales y resultados con nuestros alumnos. De todas las posibilidades que esta plataforma nos ofrece, hemos querido explotar en este caso y con fines didácticos, una opción en concreto, que hasta el momento no habíamos utilizado en todas sus posibilidades. Nos referimos al apartado de Tareas. Esta opción nos permite intercambiar archivos de sonido con nuestros alumnos enriqueciendo de forma individualizada las técnicas metodológicas de evaluación.

\section{Desarrollo de la innovación}

Pasamos pues a describir las distintas fases del proceso por el cual accedemos a la producción de nuestros alumnos de forma individualizada y cómo planteamos dos maneras diferentes de retroalimentación. Estos alumnos son estudiantes en tres centros distintos de la UPV: Escuela Técnica Superior de Ingeniería Agronómica y del Medio Natural (ETSIAMN), Escuela Técnica Superior de Ingenieros Industriales (ETSII) y Facultad de Administración y Dirección de Empresas (FADE).

En primer lugar, consideramos muy importante el hecho de que estas grabaciones fueran textos y diálogos para practicar la fonética de forma contextualizada. Tradicionalmente la instrucción de la fonética en los libros de texto para aprendizaje de una lengua extranjera, 
se ha limitado a centrar la atención sobre los sonidos y fonemas, es decir, la práctica ha estado dirigida segmentalmente y sustentada a menudo desde una concepción contrastiva del aprendizaje de segundas y terceras lenguas, como señala Gil Vádés (2010) . Sin embargo, por nuestra parte consideramos más relevante y eficaz contextualizar estas articulaciones, es decir, nos inclinamos por una práctica suprasegmental. Esta contextualización tiene lugar gracias al trabajo de los alumnos, a los que antes de la grabación, hemos pedido la producción o modificación de un texto escrito y de un diálogo. Estas dos entregas tienen lugar en dos momentos puntuales del cuatrimestre, siguiendo la progresión de la planificación en la consecución de los objetivos de la asignatura. La producción del texto y del diálogo implican un trabajo que trae consigo, no sólo la comprensión lectora y el procesamiento de la información, sino también una parte activa que está relacionada con aspectos gramaticales de la lengua meta. Es también relevante señalar que en el caso del texto se trata de un trabajo individual; y en el del diálogo de una actividad por parejas.

Esta actividad se desarrolla en tres fases:

a) El alumno graba su voz y sube el archivo a PoliformaT (espacio compartido o Tarea). Puede subir la mejor obtenida según su parecer (sentido autocrítico) o sube dos versiones: la primera y la mejorada.

b) El profesor escucha la grabación (o las grabaciones) y las valora.

c) El alumno recibe la valoración del profesor, bien en la corrección de la misma Tarea o bien en el aula (dependiendo del tamaño del grupo).

En la Figura 1 puede observarse un ejemplo de un envío de Tarea con dos archivos de sonido y los comentarios del profesor para mejorar la pronunciación, indicando los errores $\mathrm{y}$ adjuntando las referencias de las palabras tal y como aparecen en un diccionario online.

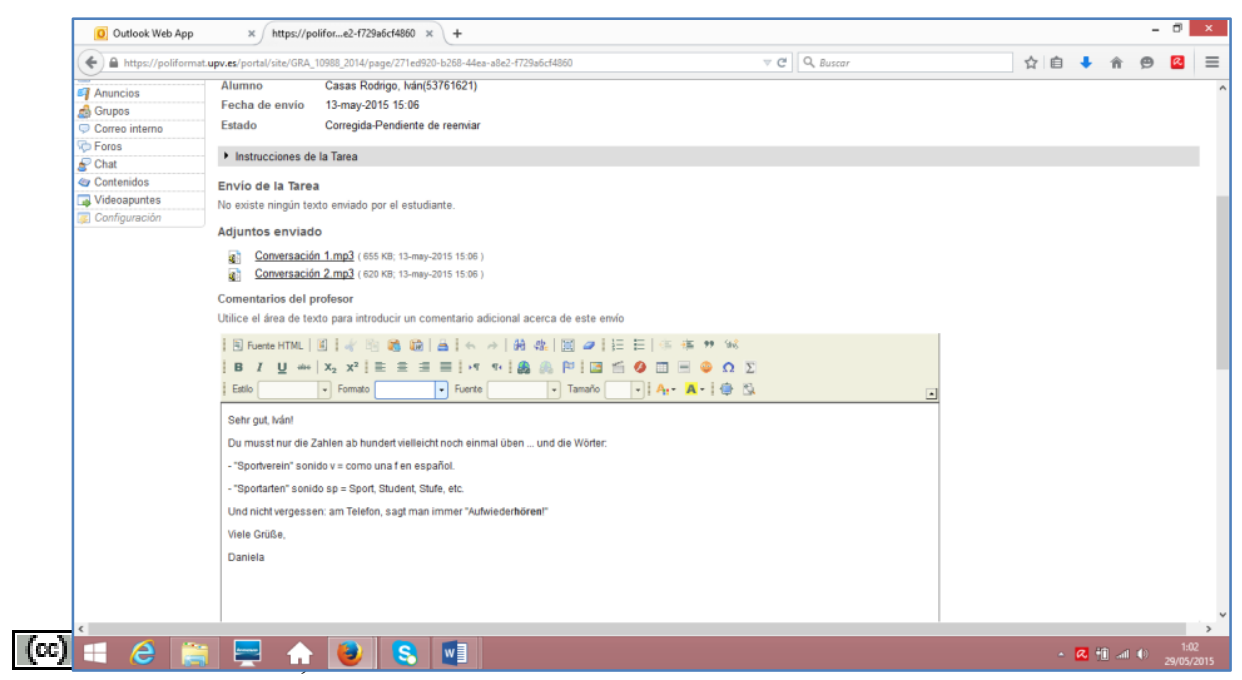

Congreso In-Red (2015) 
Fig. 1 Ejemplo de envío y corrección

\section{Resultados}

Los resultados estrictamente lingüísticos, nos referimos a una buena pronunciación, pueden estar influidos por diversos procesos, como por ejemplo, la asistencia a clase, la exposición a la lengua meta fuera del aula, la práctica continuada con nativos, el visionado de películas, la audición de canciones, etc. Pero lo que realmente favorece una pronunciación y entonación correctas es sin duda la práctica y su (auto)evaluación. De ahí la importancia de escucharse a sí mismos. Pero, ¿están de acuerdo con esta afirmación nuestros alumnos?, ¿son ellos mismos críticos a la hora de escoger el método más adecuado para practicar y aprender?, ¿utilizan todos el mismo?. Con objeto de conocer sus percepciones, elaboramos una encuesta, cuyos resultados recogemos a continuación.

En la ETSIAMN respondieron a la encuesta 8 alumnos,en la ETSII 20 y en FADE 15 alumnos. Las cuestiones planteadas fueron las siguientes:

1. ¿Cuántas veces has consultado la web de fonética que hemos recomendado en el aula?

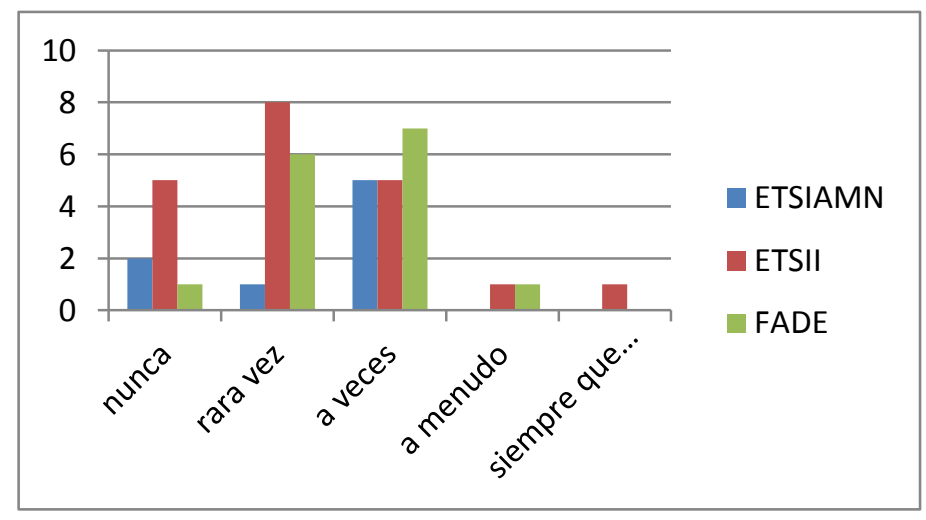

Fig. 2 Resultados Pregunta 1

En la Figura 2 queda reflejado el interés por consultar una web especializada para Fonética. Las respuestas cercanas a un uso puntual son las mayoritarias y son bastante homogéneas a pesar de ser respondidas en tres centros distintos. 
2. ¿Cuántas veces has usado el diccionario PONS/LEO para escuchar la pronunciación de una palabra?

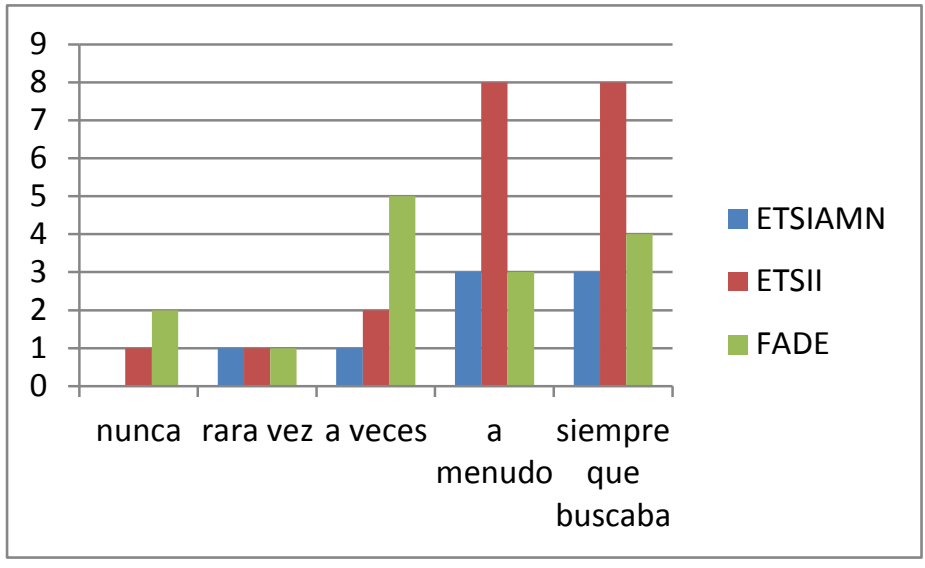

Fig. 3 Resultados Pregunta 2

Es en la pregunta 2, reflejada en la Figura 3, donde coinciden la mayoría de nuestros alumnos en la utilidad de los diccionarios online para consultar la pronunciación. Este resultado contrasta con el de la pregunta 3 (Figura 4) el cual refleja un uso diversificado del CD del libro de texto.

3. ¿Cuántas veces has escuchado las pistas de audio desde el CD del libro de texto? 


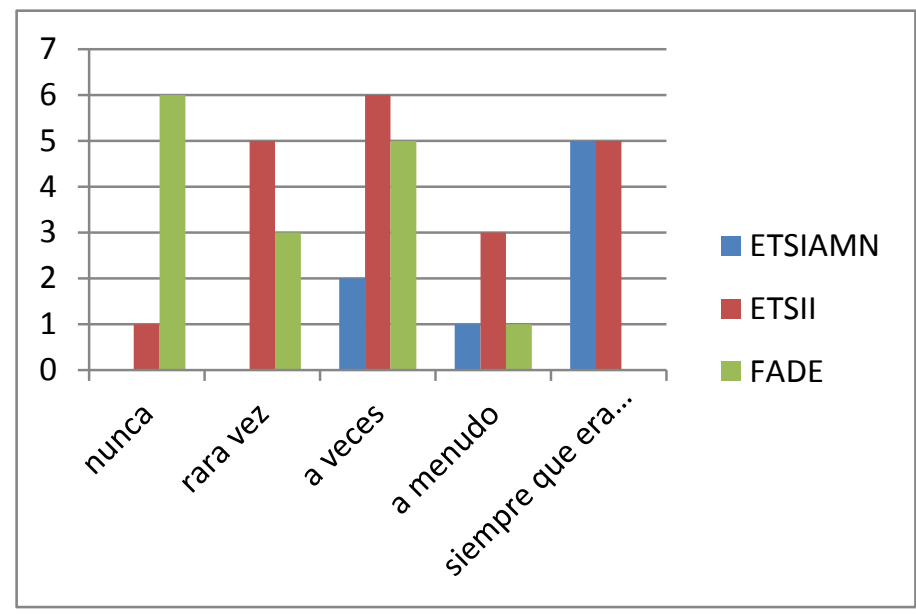

Fig. 4 Resultados Pregunta 3

Escuchar las pistas desde el CD del libro parece ser una práctica escogida para algunos alumnos independientemente del grupo o centro al que pertenecen.

Y, por ultimo, respecto a la frecuencia necesaria para obtener una grabación satisfactoria, se observa en la Figura 5 que esta técnica depende de la necesidad de cada discente. Si bien parece observarse que se dan distintos grados de necesidad.

4. ¿Cuántas veces grababas y escuchabas tus audios antes de subirlos a tu carpeta de Espacio Compartido?

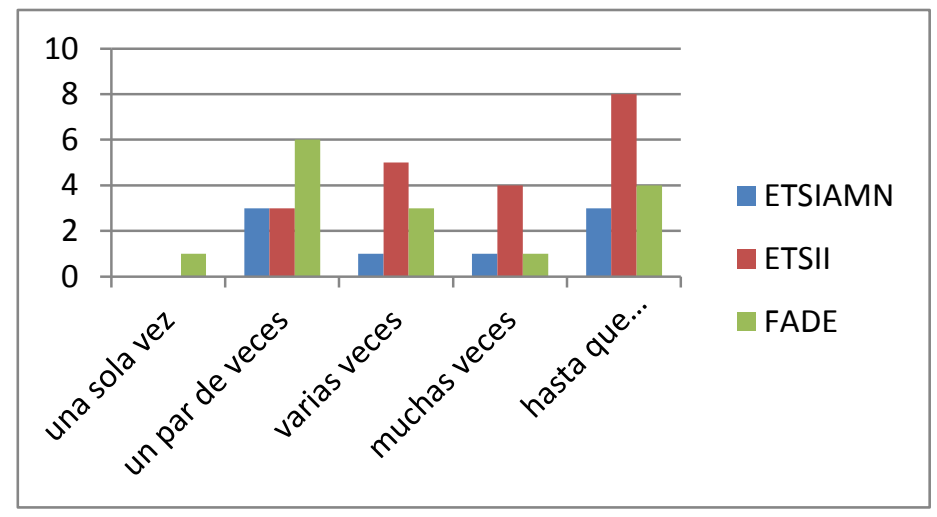

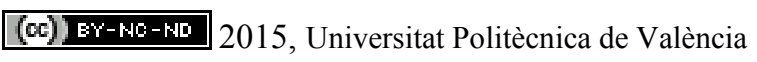


Fig. 5 Resultados pregunta 4

5. ¿Crees que tus grabaciones han servido para mejorar tu pronunciación/entonación?

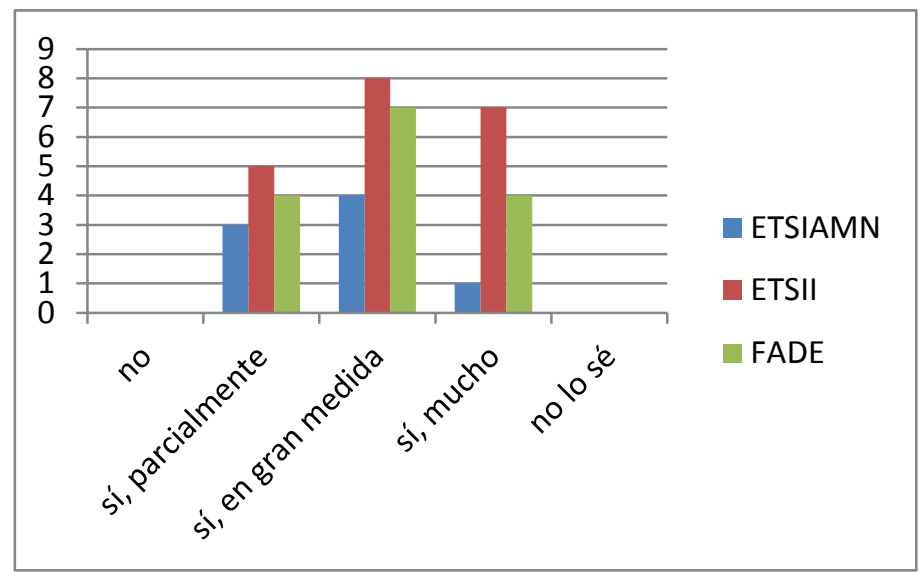

Fig. 6 Resultados Pregunta 5

Las respuestas a la cuestión 5 demuestran una concienciación del proceso de aprendizaje, puesto que no hay respuesta dubitativa. Y además, reflejan la acogida positiva de esta práctica.

6. Valora de 1 a 5 ( siendo 1 el valor mínimo y 5 el máximo) en qué medida te han ayudado los siguientes recursos/acciones para mejorar tu expresión oral en alemán:

Tabla 1. Valoración recursos

\begin{tabular}{|l|l|l|l|l|l|l|l|l|l|l|l|l|l|l|l|l|l|}
\hline & \multicolumn{3}{|l|}{ ETSIAMN } & \multicolumn{3}{|l|}{ ETSII } & \multicolumn{3}{|l|}{ FADE } \\
\hline Valoración & $\mathbf{1}$ & $\mathbf{2}$ & $\mathbf{3}$ & $\mathbf{4}$ & $\mathbf{5}$ & & $\mathbf{1}$ & $\mathbf{2}$ & $\mathbf{3}$ & $\mathbf{4}$ & $\mathbf{5}$ & & $\mathbf{1}$ & $\mathbf{2}$ & $\mathbf{3}$ & $\mathbf{4}$ & $\mathbf{5}$ \\
\hline $\begin{array}{l}\text { Web } \\
\text { fonética }\end{array}$ & 2 & 2 & 3 & 1 & 0 & & 7 & 6 & 3 & 2 & 2 & & 3 & 5 & 5 & 2 & 0 \\
\hline
\end{tabular}

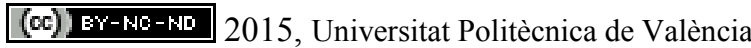




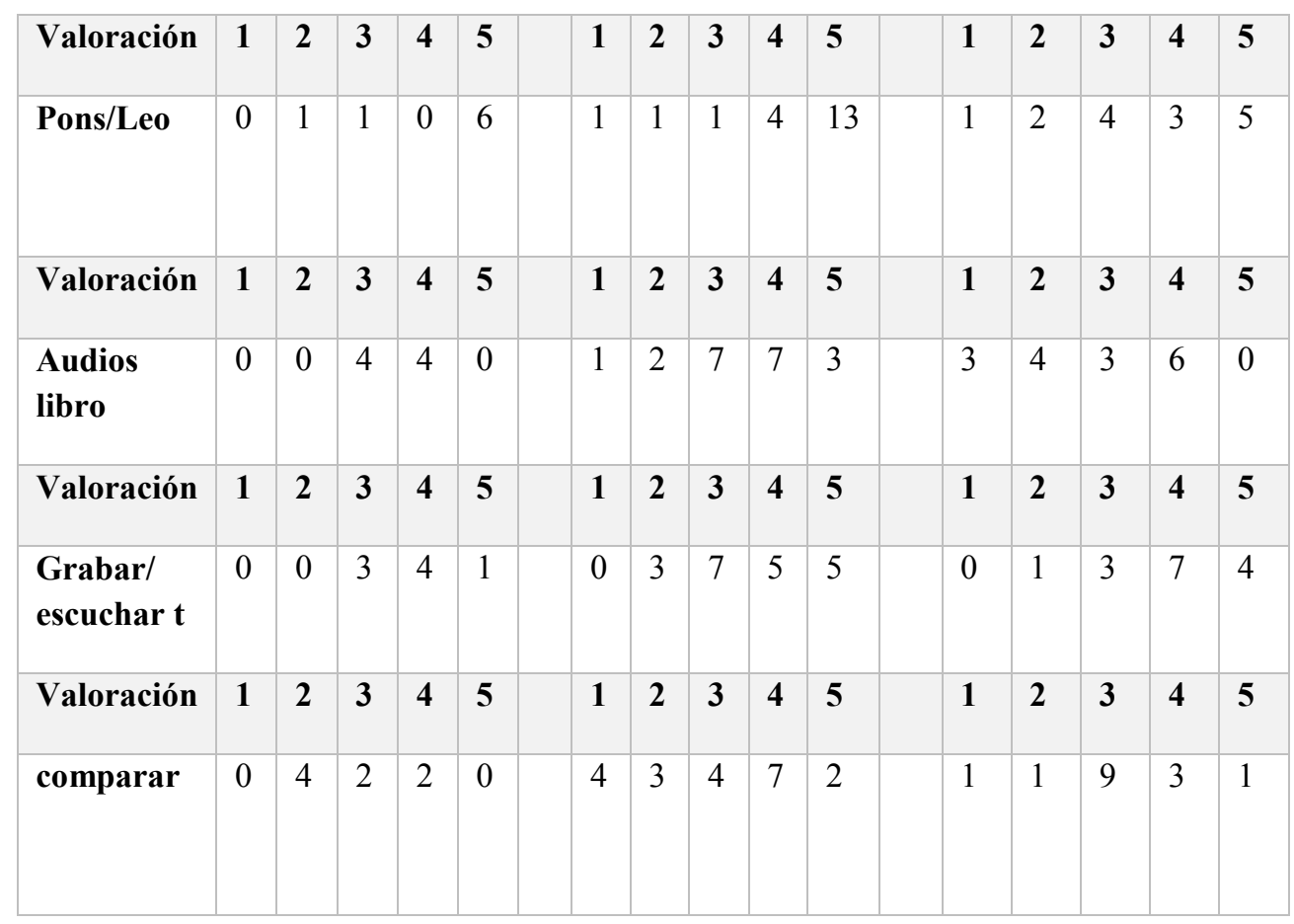

En cuanto a la valoración de los distintos recursos o acciones para mejorar, observamos unanimidad en valorar muy positivamente la consulta en diccionarios online. A continuación parecen también coincidir en el aprovechamiento de grabar y escuchar sus propias grabaciones. Sin embargo, quedan más repartidas las opiniones, tanto de la web de fonética, como de los audios del libro y de la comparación de sus grabaciones con los modelos.

Respecto a las posibles razones por las que se decantan los alumnos en general a valorar más positivamente la consulta en los diccionarios online, pensamos que pueda deberse a la comodidad de hallar en una misma pantalla información acerca del significado, del género, de la forma del plural, transcripción fonética y sonido de la palabra consultada. El sistema es rápido y eficaz.

Grabarse y escucharse a sí mismos también es percibido como provechoso, puesto que la mayoría de los estudiantes ha valorado este recurso a partir de 3 .

\section{Conclusiones}


La motivación es un elemento fundamental para el éxito en el aprendizaje de una segunda lengua o lengua extranjera (Corder 1991; Castrillo 2013). Hemos comprobado que los alumnos se sienten motivados al poder oír su propia voz en otro idioma. Las grabaciones que hemos puesto en práctica les han permitido ser protagonistas de su propio aprendizaje pudiendo escucharse tantas veces como fuera necesario y escuchar a su vez los textos orales de referencia con un objetivo definido y por lo tanto significativo. En palabras de Farrús et al. (2011:42) "d'aquesta manera, els estudiants es converteixen en els autèntics protagonistas de l'activitat, i el professor en un observador que reacciona i intervé quan l'estudiant ho necessita".

En la descripción del desarrollo de nuestra innovación hemos evidenciado cómo ha sido fomentado el aprendizaje autónomo y la capacidad de autocrítica, valores estos que ha de potenciar todo docente, así como la importancia de aprender de los propios errores por parte del discente, dándole un valor indispensable a la interlengua que surge en el proceso de aprendizaje de la lengua extranjera. En palabras de Fonseca (2007:9) "los errores ya no son vistos como algo a ser evitado a toda costa, sino como un reflejo de los procesos y estrategias de los aprendices, es decir, como una manifestación de una competencia en evolución. “

Consideramos, pues, que este trabajo muestra que las herramientas tecnológicas implementan el proceso de aprendizaje, en nuestro caso concreto, la adquisición de la expresión oral en lengua alemana stándard, sin olvidar que siempre han de ir acompañadas de unos criterios metológicos justificados en la literatura científica correspondiente.

\section{Referencias}

CABEDO NEBOT, A. (2012). „Actividades de articulación y de percepción auditiva: uso de herramientas informáticas" en Foro de profesores de E/LE, 8, p. 1-12.

CASTRILLO DE LARRETA-AZELAIN (2013). "Learners attitudes toward collaborative writing in e-learning clases: a twitter Project for German as a foreign language" en RESLA, 26, p.127-138.

CORDER, S.P. (1991). "La importancia de los errores del que aprende una lengua segunda" en Muñoz Liceras, J. La adquisición de las Lenguas extranjeras. Madrid: Visor. FARRÚS, M. et al. (2011). "L'ensenyament universitari del segle XXI: més enllà del paper". En Item 55, p. 33-47.

FONSECA OLIVEIRA, A. (2007). "Análisis de la Interlengua fónica" en Phónica, 3-31.

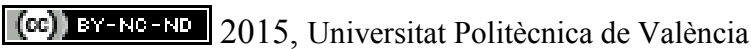


GIL VALDÉS, M.J. (2010). "Transferencias positiva y negativa en la adquisición de la fonética alemana por estudiantes españoles" en Cuadernos de Filología alemana, anejo II, 109-115.

HIRSCHFELD, U. y WOLFF, J. (2007). Nicht auf dem Mund gefallen. Madrid: Editorial Idiomas.

HIRSCHFELD, U. y REINKE, K. (2007). „Phonetik in Deutsch als Fremdsprache: Theorie und Praxis - Einführung in das Themenheft" en Zeitschrift für Interkulturellen Fremdsprachenunterricht. 12, 2, 1-7.

MINISTERIO DE EDUCACIÓN, CULTURA Y DEPORTE, SUBDIRECCIÓN GENERAL DE COOPERACIÓN INTERNACIONAL. Marco común europeo de referencia para las lenguas: aprendizaje, enseñanza, evaluación.

$<\mathrm{http}: / /$ cvc.cervantes.es/ensenanza/biblioteca_ele/marco/cvc_mer.pdf $>$ [20 de mayo de 2015]

MEBUS, G. (1995). „Erfolgskontrolle, Prüfung, Bewertung- auch für die Aussprache?“ en Fremdsprache Deutsch, 12, p. 26-30.

PANUŠOVÁ, M. (2007). "Phonetik in DaF-Lehrwerken" en Zeitschrift für Interkulturellen Fremdsprachenunterricht. 12,2, p. 1-22.

PASTOR CESTEROS, S. (2004). Aprendizaje de segundas lenguas. Lingüística aplicada a la enseñanza de idiomas. Alicante: Universidad de Alicante. 